\title{
Precision calculation of above-threshold multiphoton ionization in intense short-wavelength laser fields: The momentum-space approach and time-dependent generalized pseudospectral method
}

\author{
Zhongyuan Zhou ${ }^{1, *}$ and Shih-I Chu ${ }^{1,2, \dagger}$ \\ ${ }^{1}$ Department of Chemistry, University of Kansas, Lawrence, Kansas 66045, USA \\ ${ }^{2}$ Center for Quantum Science and Engineering, Department of Physics, National Taiwan University, Taipei 10617, Taiwan
}

(Received 13 November 2010; published 19 January 2011)

\begin{abstract}
We present an approach in momentum $(\mathcal{P})$ space for the accurate study of multiphoton and above-threshold ionization (ATI) dynamics of atomic systems driven by intense laser fields. In this approach, the electron wave function is calculated by solving the $\mathcal{P}$-space time-dependent Schrödinger equation (TDSE) in a finite $\mathcal{P}$-space volume under a simple zero asymptotic boundary condition. The $\mathcal{P}$-space TDSE is propagated accurately and efficiently by means of the time-dependent generalized pseudospectral method with optimal momentum grid discretization and a split-operator time propagator in the energy representation. The differential ionization probabilities are calculated directly from the continuum-state wave function obtained by projecting the total electron wave function onto the continuum-state subspace using the projection operator constructed by the continuum eigenfunctions of the unperturbed Hamiltonian. As a case study, we apply this approach to the nonperturbative study of the multiphoton and ATI dynamics of a hydrogen atom exposed to intense shortwavelength laser fields. High-resolution photoelectron energy-angular distribution and ATI spectra have been obtained. We find that with the increase of the laser intensity, the photoelectron energy-angular distribution changes from circular to dumbbell shaped and is squeezed along the laser field direction. We also explore the change of the maximum photoelectron energy with laser intensity and strong-field atomic stabilization phenomenon in detail.
\end{abstract}

DOI: 10.1103/PhysRevA.83.013405

PACS number(s): 32.80.Rm, 32.80.Fb, 33.80.Rv

\section{INTRODUCTION}

Multiphoton and above-threshold ionization (ATI) of an atom exposed to intense laser fields has been an attractive topic for decades [1,2] since it is first discovered in 1979 [3]. Owing to the advance of the intense and short pulse laser technology, the study of ATI phenomenon continues to attract much attention [4,5]. The multiphoton ATI can be attributed to two distinct regimes based on the Keldysh parameter [6], $\gamma=\sqrt{I_{P} / 2 U_{P}}$, where $I_{P}$ is the ionization potential of the atom, $U_{P}=\varepsilon_{0}^{2} / 4 \omega^{2}$ (atomic units are used throughout this paper unless otherwise indicated) is the ponderomotive energy, $\varepsilon_{0}$ is the amplitude of the laser field, and $\omega$ is the laser angular frequency. For a fixed laser frequency $\omega$, in the weak laser fields when $\gamma \gg 1$, the multiphoton absorption is the dominating mechanism for the ATI, while in the intense laser fields when $\gamma \ll 1$, the tunneling ionization is the primary mechanism. At the transition region when $\gamma \approx 1$, both the multiphoton ionization and tunneling ionization contribute to the ATI. The photoelectrons induced directly by the laser fields have a classical cutoff energy $2 U_{P}$, while the electrons produced by the rescattering can extend to the maximum energy up to $10 U_{P}[7-10]$.

Extensive investigations have been performed both theoretically [11-18] and experimentally [7,12,14,19-31] for a comprehensive understanding of the laser-induced atomic ATI for laser fields with wavelengths from visible lights $\left(4 \times 10^{2} \mathrm{~nm}\right)$ to infrared radiations $\left(1 \times 10^{5} \mathrm{~nm}\right)$. With the recent development of intense and ultrashort-wavelength free-electron lasers [32-34], the study of multiphoton

\footnotetext{
*zyzhou@ku.edu

†sichu@ku.edu
}

processes in the high-frequency and strong-field regime becomes increasingly important [35-37]. In such a regime, the electron can achieve very high energy by absorbing photons from the fields and can go very far from the nucleus. Likewise, the electron wave function will extend to a very large distance, even to infinity. To solve the time-dependent Schrödinger equation (TDSE) in spatial coordinate $(\mathcal{R})$ space, the boundary has to be set at a very large distance to avoid the reflection of the wave function. This will require the use of a very large number of grid points to cover a large range of spatial space, rendering the $\mathcal{R}$-space calculation difficult to perform accurately and efficiently.

The difficulty in the $\mathcal{R}$-space numerical calculation may be overcome by the computation in momentum $(\mathcal{P})$ space instead. In any physical process, the momentum of an electron is always finite and less than a certain maximum value $k_{\max }$. The probability of electron is negligible or zero when its momentum is greater than $k_{\max }$. Thus, in the $\mathcal{P}$ space, the wave function of the electron is localized (e.g., the wave function of a free electron is a $\delta$ function in the $\mathcal{P}$ space) and can be calculated in a finite $\mathcal{P}$-space volume with a simple zero boundary condition as long as the boundary of the volume is set at the place with a properly large momentum $k_{\max }$. This ensures that all the information with regard to the continuum-state physical processes are included in the electron wave function, and at the same time makes the computation simple and efficient.

The $\mathcal{P}$-space approach has been considered in the past for several different time-dependent problems, including, for example, atomic photoionization [38-40] and electron quantum transport in the molecular device [41]. Most of these earlier studies were performed for the one-dimensional systems, apart from the recent calculation [40]. This calculation that used the modified kernel was confined in a finite-sized 
$\mathcal{R}$-space box with a small value of $k_{\max }$, and it could predict the main features of the ATI spectra for the hydrogen atom in intense laser fields. However, for precise calculation and/or intense short-wavelength laser fields, both the size of the box and the value of $k_{\max }$ have to be increased to avoid the wave function reflection on the boundary and to calculate the ATI spectra at high energy.

In this paper, we present an efficient computational approach in the $\mathcal{P}$ space for the accurate study of multiphoton processes of atomic systems driven by intense laser fields. This approach is free of boundary reflection because the basic equation of the approach is the $\mathcal{P}$-space TDSE that is obtained from the Fourier transform of the $\mathcal{R}$-space TDSE in the whole $\mathcal{R}$ space. The electron wave function is calculated within a finite $\mathcal{P}$-space volume under a simple zero boundary condition by solving the $\mathcal{P}$-space TDSE. The $\mathcal{P}$-space TDSE is propagated accurately and efficiently by means of the time-dependent generalized pseudospectral (TDGPS) method together with the optimal momentum discretization and a split-operator time propagator in the energy representation [42-45]. The differential ionization probabilities are calculated directly from the total electron wave function at the end of the laser field with the help of a continuum-state projection operator. The continuumstate projection operator is constructed by the continuum eigenfunctions of the unperturbed Hamiltonian. The proposed approach has been applied to the accurate study of multiphoton ATI processes of the hydrogen atom driven by intense shortwavelength laser fields where the $\mathcal{R}$-space methods cannot work efficiently and effectively. The high-resolution photoelectron energy-angular distribution and ATI spectra have been obtained. The changes of the energy-angular distribution, ATI spectra, the maximum photoelectron energy, and total ionization probability with laser intensity have been explored in detail. Some distinctive phenomena have been predicted.

\section{THEORETICAL AND NUMERICAL METHODOLOGY}

\section{A. $\mathcal{P}$-space time-dependent Schrödinger equation}

In the velocity gauge, the $\mathcal{R}$-space TDSE in the dipole approximation for an atom interacting with a laser field is given by

$$
i \frac{\partial}{\partial t} \Psi(\mathbf{r}, t)=\left[\frac{\widehat{\mathbf{p}}^{2}}{2}+\frac{1}{c} \mathbf{A}(t) \cdot \widehat{\mathbf{p}}+U(\mathbf{r})\right] \Psi(\mathbf{r}, t),
$$

where $c$ is the light speed, $\mathbf{A}(t)$ is the vector potential of the laser field, and $U(\mathbf{r})$ is the Coulomb potential of the atom. The $\mathcal{P}$-space wave function $\Phi(\mathbf{k}, t)$ can be calculated from the Fourier transform of the $\mathcal{R}$-space wave function $\Psi(\mathbf{r}, t)$ in the whole $\mathcal{R}$ space,

$$
\Phi(\mathbf{k}, t)=\frac{1}{(2 \pi)^{3 / 2}} \int \Psi(\mathbf{r}, t) \exp (-i \mathbf{k} \cdot \mathbf{r}) d \mathbf{r} .
$$

Applying Eq. (2) to (1), one obtains the $\mathcal{P}$-space TDSE for the $\mathcal{P}$-space wave function $\Phi(\mathbf{k}, t)$, which is an integro-differential equation given by

$$
\begin{aligned}
i \frac{\partial}{\partial t} \Phi(\mathbf{k}, t)= & \frac{\mathbf{k}^{2}}{2} \Phi(\mathbf{k}, t)+\frac{1}{c} \mathbf{A}(t) \cdot \mathbf{k} \Phi(\mathbf{k}, t) \\
& +\int V\left(\mathbf{k}, \mathbf{k}^{\prime}\right) \Phi\left(\mathbf{k}^{\prime}, t\right) d \mathbf{k}^{\prime},
\end{aligned}
$$

where, $V\left(\mathbf{k}, \mathbf{k}^{\prime}\right)$ is the $\mathcal{P}$-space Coulomb potential defined by

$$
V\left(\mathbf{k}, \mathbf{k}^{\prime}\right)=\frac{1}{(2 \pi)^{3}} \int U(\mathbf{r}) \exp \left[i\left(\mathbf{k}^{\prime}-\mathbf{k}\right) \cdot \mathbf{r}\right] d \mathbf{r} .
$$

For a hydrogenic atom, the $\mathcal{P}$-space Coulomb potential is given by $[46,47]$

$$
V\left(\mathbf{k}, \mathbf{k}^{\prime}\right)=-\frac{Z}{2 \pi^{2}} \frac{1}{\left|\mathbf{k}-\mathbf{k}^{\prime}\right|^{2}} .
$$

Because the integral in Eq. (2) is over the whole $\mathcal{R}$ space, the $\mathcal{P}$-space wave function contains all the information of the electron in physical processes of the whole $\mathcal{R}$ space and the $\mathcal{P}$-space calculation does not suffer from the reflection of the wave function on the boundary.

\section{B. Partial wave expansion and radial wave function}

Assume that a hydrogenic atom in the ground state is exposed to the laser field along $z$ axis. In this case, the system is axially symmetric about the $z$ axis during evolution, and the wave function $\Phi(\mathbf{k}, t)$ can be expanded in the partial waves as

$$
\Phi(\mathbf{k}, t)=\frac{1}{k} \sum_{l=0}^{l_{\max }} \varphi_{l}(k, t) Y_{l 0}(\theta, \phi),
$$

where $l_{\max }$ is the maximum number of the partial waves, $\varphi_{l}(k, t)$ is the radial wave function, and $Y_{l m}(\theta, \phi)$ is the spherical harmonic. For the hydrogenic atom, the Coulomb potential Eq. (5) can also be expanded in the partial waves as [46-48]

$$
V\left(\mathbf{k}, \mathbf{k}^{\prime}\right)=\frac{1}{k k^{\prime}} \sum_{l=0}^{l_{\max }} \sum_{m=-l}^{l} V_{l}\left(k, k^{\prime}\right) Y_{l m}(\theta, \phi) Y_{l m}^{*}\left(\theta^{\prime}, \phi^{\prime}\right),
$$

where $k=|\mathbf{k}|$ and

$$
V_{l}\left(k, k^{\prime}\right)=-\frac{Z}{\pi} Q_{l}\left(\frac{k^{2}+k^{\prime 2}}{2 k k^{\prime}}\right) .
$$

Here $Q_{l}(z)$ is the Legendre function of the second kind.

Applying Eqs. (6) and (7) to (3), we obtain an integrodifferential equation for the $\mathcal{P}$-space radial wave function $\varphi_{l}(k, t)$

$$
\begin{aligned}
i \frac{\partial}{\partial t} \varphi_{l}(k, t)= & \frac{k^{2}}{2} \varphi_{l}(k, t)+\int V_{l}\left(k, k^{\prime}\right) \varphi_{l}\left(k^{\prime}, t\right) d k^{\prime} \\
& +\frac{1}{c} k A(t)\left[\alpha_{l+1} \varphi_{l+1}(k, t)+\alpha_{l} \varphi_{l-1}(k, t)\right],
\end{aligned}
$$

where

$$
\alpha_{l}=\frac{l}{\sqrt{(2 l-1)(2 l+1)}} .
$$

\section{Landé subtraction technique}

The partial wave form of the $\mathcal{P}$-space Coulomb potential $V_{l}\left(k, k^{\prime}\right)$ has a logarithmic singularity at $k=k^{\prime}$ because the Legendre function of the second kind is logarithmically singular at $k=k^{\prime}$. This singularity makes it difficult to accurately calculate the wave function and is one of the most troublesome issues for numerically solving the $\mathcal{P}$-space Schrödinger equation. To remove this singularity, the Landé subtraction technique has been proposed [49]. Following this 
technique, the term involving Coulomb potential in Eq. (9) is calculated by

$$
\begin{aligned}
& \int V_{l}\left(k, k^{\prime}\right) \varphi_{l}\left(k^{\prime}, t\right) d k^{\prime} \\
& =k S_{l} \varphi_{l}(k, t)+\int V_{l}\left(k, k^{\prime}\right)\left[\varphi_{l}\left(k^{\prime}, t\right)-\frac{\varphi_{l}(k, t)}{P_{l}(z)} \frac{k}{k^{\prime}}\right] d k^{\prime},
\end{aligned}
$$

where $z=\left(k^{2}+k^{\prime 2}\right) / 2 k k^{\prime}, P_{l}(z)$ is the Legendre polynomial, and $S_{l}$ is defined by

$$
S_{l}=\int \frac{V_{l}\left(k, k^{\prime}\right)}{P_{l}(z)} \frac{d k^{\prime}}{k^{\prime}},
$$

and can be calculated numerically [50]. In Eq. (11), the terms in the square bracket tend to zero faster than the Coulomb potential $V_{l}\left(k, k^{\prime}\right)$ tends to infinite when $k^{\prime} \rightarrow k$. Thus the integral is zero at $k^{\prime}=k$ and the singularity is removed. The Landé subtraction technique has been applied to the hydrogen atom [46-48] and produced quite accurate eigenvalues and eigenfunctions for any angular momentum [48] compared to the analytical ones [51]. The Landé subtraction technique has also been applied successfully to the numerical calculations of relativistic Klein-Gordon equations [49], Dirac equations [49], and Bethe-Salpeter equations [47,52].

For further simplification, we introduce

$$
v_{l}\left(k, k^{\prime}\right)= \begin{cases}0, & k^{\prime}=k, \\ V_{l}\left(k, k^{\prime}\right), & k^{\prime} \neq k .\end{cases}
$$

Substituting Eq. (11) into Eq. (9) and using Eq. (13), we gain a singularity-free equation for the $\mathcal{P}$-space radial wave function $\varphi_{l}(k, t)$,

$$
\begin{aligned}
i \frac{\partial}{\partial t} \varphi_{l}(k, t)= & {\left[\frac{k^{2}}{2}+k S_{l}-k q_{l}(k)\right] \varphi_{l}(k, t) } \\
& +\int v_{l}\left(k, k^{\prime}\right) \varphi_{l}\left(k^{\prime}, t\right) d k^{\prime} \\
& +\frac{1}{c} k A(t)\left[\alpha_{l+1} \varphi_{l+1}(k, t)+\alpha_{l} \varphi_{l-1}(k, t)\right]
\end{aligned}
$$

where

$$
q_{l}(k)=\int \frac{v_{l}\left(k, k^{\prime}\right)}{P_{l}(z)} \frac{d k^{\prime}}{k^{\prime}} .
$$

\section{Generalized pseudospectral method}

Because the electron momentum is always finite and the $\mathcal{P}$-space electron wave function can be set to zero on the boundary with a sufficiently large value of $k_{\max }$, we will confine our calculation in the volume of $k \in\left[0, k_{\max }\right]$. To solve Eq. (14), we extend the generalized pseudospectral (GPS) method [42-45] to the $\mathcal{P}$-space calculation. We first map the $\mathcal{P}$-space domain $k \in\left[0, k_{\text {max }}\right]$ to a new domain $x \in[-1,1]$ by a mapping function

$$
k(x)=\gamma \frac{1+x}{1-x+x_{m}},
$$

where $\gamma$ is the mapping parameter and $x_{m}=2 \gamma / k_{\max }$. The smaller the value of $\gamma$, the denser the grid points at small $k$. By changing the mapping parameter, the grid points can be adjusted in some way so as to improve the calculation of the wave function. On the new domain $x \in[-1,1]$, Eq. (14) is discretized by using the Gaussian quadrature with $N$ grid points $\left(x_{1}, x_{2}, \ldots, x_{N}\right)$ and is converted to a time-dependent symmetric matrix equation

$$
i \frac{\partial}{\partial t} y_{l}^{j}(t)=\sum_{j^{\prime}=1}^{N} h_{l}^{j j^{\prime}} y_{l}^{j^{\prime}}(t)+\sum_{l^{\prime}=0}^{l_{\max }} \bar{h}_{l l^{\prime}}^{j} y_{l^{\prime}}^{j}(t),
$$

where

$$
\begin{gathered}
y_{l}^{j}(t)=\sqrt{\dot{k}_{j} w_{j}} \varphi_{l}^{j}(t), \\
h_{l}^{j j^{\prime}}=\left(\frac{k_{j}^{2}}{2}+k_{j} S_{l}-k_{j} q_{l}^{j}\right) \delta_{j j^{\prime}}+v_{l}^{j j^{\prime}} \sqrt{w_{j} \dot{k}_{j} w_{j^{\prime}} \dot{k}_{j^{\prime}}}, \\
\bar{h}_{l l^{\prime}}^{j}=\frac{1}{c} k_{j} A(t)\left(\alpha_{l+1} \delta_{l+1 l^{\prime}}+\alpha_{l} \delta_{l-1 l^{\prime}}\right) .
\end{gathered}
$$

Here, $\quad k_{j}=k\left(x_{j}\right), \quad \varphi_{l}^{j}(t)=\varphi_{l}\left(k_{j}, t\right), \quad q_{l}^{j}=q_{l}\left(k_{j}\right), \quad v_{l}^{j j^{\prime}}=$ $v_{l}\left(k_{j}, k_{j^{\prime}}\right), \quad \dot{k}_{j^{\prime}}=d k /\left.d x\right|_{x_{j^{\prime}}}, \quad$ and $w_{j} \quad$ is the weight of the Gaussian quadrature, $h_{l}^{j j^{\prime}}$ is the unperturbed Hamiltonian matrix element, and $\bar{h}_{l l^{\prime}}^{j}$ is the interaction matrix element. The GPS method associated with the mapping technique allows for nonuniform and optimal discretization with the use of only a modest number of grid points. It has been shown that the GPS method is a very effective and efficient numerical algorithm for the high-precision solution of the Schrödinger equation and has been widely applied to the $\mathcal{R}$-space calculations with Coulomb potentials [42-45].

The wave function can be achieved by numerical calculation of the time-dependent matrix equation (17) step by step in time by using the second-order split-operator method in the energy representation [44]. Because the unperturbed Hamiltonian matrix in the first term on the right-hand side of Eq. (17), $\mathbf{H}_{0}=\left\{h_{l}^{j j^{\prime}} \delta_{l l^{\prime}}\right\}$, and the laser-atom interaction matrix in the second term, $\mathbf{H}_{1}=\left\{\bar{h}_{l l^{\prime}}^{j} \delta_{j j^{\prime}}\right\}$, are diagonal with respect to $l$ and $j$, respectively, the propagation of the wave function is constrained in the subspace with the same $l$ for the first term and the subspace with the same $j$ for the second term. This will save computer resources (CPU time and memory) and speed up the wave function time propagation.

\section{E. Differential ionization probability and photoelectron spectra}

In the intense laser field, an electron can be ionized by absorbing energy from the laser field directly and/or gaining the energy from rescattered electrons. The differential ionization probabilities and photoelectron energy-angular distribution can be extracted from the wave function $\Phi\left(\mathbf{k}, t_{f}\right)$ at the end of the laser field. The conventional method for the calculation of these quantities is to project the wave function to the continuum states constructed by the Coulomb wave function [53,54]. This method can also be extended to the $\mathcal{P}$-space calculation of the differential ionization probabilities [55]. However, just as the $\mathcal{R}$-space wave function represents the probability amplitude of an electron at the spatial position $\mathbf{r}$, the $\mathcal{P}$-space wave function characterizes the probability amplitude of an electron having the momentum $\mathbf{k}$ and kinetic energy $k^{2} / 2$. Thus the $\mathcal{P}$-space wave function itself offers a very effective method for direct calculation of the differential ionization probability. The key 
point is how to extract the continuum-state wave function from the total electron wave function.

To isolate the bound-state and continuum-state wave functions from the total wave function, we introduce a bound-state projection operator by

$$
\widehat{P}_{B}=\sum_{\text {all } E_{\alpha}<0}\left|\psi_{\alpha}\right\rangle\left\langle\psi_{\alpha}\right|,
$$

and a continuum-state projection operator by

$$
\widehat{P}_{C}=\sum_{\text {all } E_{\alpha} \geqslant 0}\left|\psi_{\alpha}\right\rangle\left\langle\psi_{\alpha}\right|,
$$

respectively, where $E_{\alpha}$ and $\left|\psi_{\alpha}\right\rangle$ are the eigenvalue and eigenfunction of the unperturbed Hamiltonian. Obviously $\widehat{P}_{B}+\widehat{P}_{C}=I$.

Using the projection operators, the total wave function $\Phi\left(\mathbf{k}, t_{f}\right)$ can be divided into two parts as

$$
\Phi\left(\mathbf{k}, t_{f}\right)=\Phi_{B}\left(\mathbf{k}, t_{f}\right)+\Phi_{C}\left(\mathbf{k}, t_{f}\right),
$$

where

$$
\Phi_{B}\left(\mathbf{k}, t_{f}\right)=\widehat{P}_{B} \Phi\left(\mathbf{k}, t_{f}\right)=\sum_{\text {all } E_{\alpha}<0} c_{\alpha}\left|\psi_{\alpha}(\mathbf{k})\right\rangle
$$

and

$$
\Phi_{C}\left(\mathbf{k}, t_{f}\right)=\widehat{P}_{C} \Phi\left(\mathbf{k}, t_{f}\right)=\sum_{\text {all } E_{\alpha} \geqslant 0} c_{\alpha}\left|\psi_{\alpha}(\mathbf{k})\right\rangle
$$

are the bound-state and continuum-state wave functions, respectively, and

$$
c_{\alpha}=\left\langle\psi_{\alpha}(\mathbf{k}) \mid \Phi\left(\mathbf{k}, t_{f}\right)\right\rangle
$$

is the coefficient.

The triple differential ionization probability, which is the probability density of an photoelectron at $\mathbf{k}=(k, \theta, \phi)$ having a kinetic energy $E=k^{2} / 2$, is computed from

$$
\frac{\partial^{3} P}{\partial E \partial \Omega}=k\left|\Phi_{C}\left(\mathbf{k}, t_{f}\right)\right|^{2},
$$

where $d \Omega=\sin \theta d \theta d \phi$. Using Eq. (23), the triple differential ionization probability can also be calculated by

$$
\begin{aligned}
\frac{\partial^{3} P}{\partial E \partial \Omega}= & k\left\{\left|\Phi\left(\mathbf{k}, t_{f}\right)\right|^{2}-\left|\Phi_{B}\left(\mathbf{k}, t_{f}\right)\right|^{2}\right. \\
& \left.-2 \operatorname{Re}\left[\Phi_{B}^{*}\left(\mathbf{k}, t_{f}\right) \Phi_{C}\left(\mathbf{k}, t_{f}\right)\right]\right\}
\end{aligned}
$$

In this equation, the first term on the right-hand side is the total probability density, the second term is the probability density of the bound-state electron, and the third term is the interference between the bound-state and continuum-state electrons. This expression can be used to study the contribution of each individual process to the ionization probability.

Because the atomic system is axially symmetric, the triple differential ionization probability is independent of $\phi$. Thus the photoelectron energy-angular distribution is described by the double differential ionization probability (DDIP)

$$
\frac{\partial^{2} P}{\partial E \sin \theta \partial \theta}=2 \pi\left(\frac{\partial^{3} P}{\partial E \partial \Omega}\right) .
$$

The photoelectron ATI spectra is characterized by the differential ionization probability (DIP)

$$
\frac{d P}{d E}=\int_{0}^{\pi}\left(\frac{\partial^{2} P}{\partial E \sin \theta \partial \theta}\right) \sin \theta d \theta .
$$

Finally, the total ionization probability (TIP) can be calculated by

$$
P=\int_{0}^{\infty}\left(\frac{d P}{d E}\right) d E .
$$

The proposed $\mathcal{P}$-space approach, together with the method for the calculation of the differential ionization probabilities, can be extended easily to many-electron atomic and molecular systems within a single-active electron approximation (SAE). In the SAE, only a single active electron responds to the laser fields while all other electrons remain unaffected [56], and the active electron moves in the Coulomb fields of nuclei and other electrons as well as laser fields. The basic equation for the active electron [56] is very similar to the TDSE given by Eq. (1) apart from additional electron-electron Hartree and exchange-correlation potentials in the Hamiltonian [45,57]. Thus the techniques and methods for numerically solving the TDSE and calculating the differential ionization probabilities depicted above can be applied directly to the many-electron systems in the presence of laser fields in the SAE.

\section{RESULTS AND DISCUSSION}

\section{A. Multiphoton ionization rates of hydrogen atom in laser fields}

The $\mathcal{P}$-space radial eigenfunction tends to $1 / k^{l+4}$ when $k \rightarrow \infty$ rather than falls off exponentially [51]. Thus to accurately calculate the eigenfunction of the electron with a smaller angular momentum $l$, such as $s$ states with $l=0$, the value of $k_{\max }$ cannot be too small. Indeed, we find that the eigenvalues and eigenfunctions with larger $l$ can be more easily and accurately calculated using smaller $k_{\max }$. This can be understood from the uncertainty principle of quantum mechanics. Higher excited states usually have larger distances and smaller momenta than the ground and lower excited states. Thus the wave functions of the higher excited states are relatively more easy to compute in $\mathcal{P}$ space than $\mathcal{R}$ space [58].

As a test of the $\mathcal{P}$-space time-dependent wave function, we calculate ionization rates of a hydrogen atom initially in the ground state exposed to intense laser fields. The laser field is turned on with a sine-squared ramp of the amplitude in 10 optical cycles and then is kept a constant. The vector potential of the laser field is given by

$$
\mathbf{A}(t)=\mathbf{e}_{z} \frac{c \varepsilon_{0}}{\omega} f(t) \sin (\omega t+\alpha),
$$

with the envelope shape factor given by

$$
f(t)= \begin{cases}\sin ^{2}\left(\frac{\pi t}{2 \tau_{R}}\right), & 0 \leqslant t \leqslant \tau_{R}, \\ 1, & t>\tau_{R},\end{cases}
$$

where $\tau_{R}=10 T_{\mathrm{oc}}, T_{\mathrm{oc}}=2 \pi / \omega$ is the optical cycle of the laser field, $\alpha$ is the carrier-envelope phase, and $\mathbf{e}_{z}$ is the unit vector of the $z$ axis. To achieve accurate eigenfunctions, especially for the states with small $l$, we take $k_{\max }$ to be $10^{4}$ a.u. Although a quite large $k_{\max }$ is adopted, it does not mean a very 
TABLE I. Ionization rates of a hydrogen atom exposed to intense laser fields with peak intensity $I_{0}=1.75 \times 10^{14} \mathrm{~W} / \mathrm{cm}^{2}$. $[x]$ denotes multiplication by $10^{x}$.

\begin{tabular}{lccc}
\hline \hline$\omega_{0}$ (a.u.) & $\begin{array}{c}\text { Present } \\
\text { work }\end{array}$ & $\begin{array}{c}\text { Floquet } \\
\text { calculation [62] }\end{array}$ & $\begin{array}{c}\mathcal{R} \text {-space } \\
\text { calculation [44] }\end{array}$ \\
\hline 0.6 & $3.14[-3]$ & $3.14[-3]$ & $3.14[-3]$ \\
0.3 & $1.62[-3]$ & $1.61[-3]$ & $1.63[-3]$ \\
0.2 & $3.49[-3]$ & $3.50[-3]$ & $3.50[-3]$ \\
\hline \hline
\end{tabular}

large number of grid points has to be used because the GPS method and mapping technique are employed in the $\mathcal{P}$-space calculation. In this calculation, the mapping parameter $\gamma=2$, and 256 grid points are employed. The survival probability on the initial state decays slowly at the beginning of the laser field and exponentially after the amplitude of the laser field becomes constant $[59,60]$. The ionization rate can be estimated from the exponential decay rate of the survival probability on the initial state [61].

In Table I, we give the ionization rates of one-photon, twophoton, and three-photon ionization for the laser peak intensity $I_{0}=1.75 \times 10^{14} \mathrm{~W} / \mathrm{cm}^{2}$ and $\alpha=0$, together with the results of the time-independent Floquet calculation [62] and $\mathcal{R}$-space TDSE calculation [44] for comparison. It is shown that our numerical results are in good agreement with those of the time-independent Floquet calculation [62] and $\mathcal{R}$-space TDSE calculation [44].

\section{B. Multiphoton above-threshold ionization of hydrogen atom in intense longer-wavelength laser fields}

For a further test, we apply the $\mathcal{P}$-space approach to the study of the multiphoton ATI of a hydrogen atom initially in the ground state exposed to a longer-wavelength sine-squared laser pulse. The vector potential of the laser field is given by Eq. (32) with the shape factor

$$
f(t)=\sin ^{2}\left(\frac{\pi t}{T}\right),
$$

where $T$ is the laser pulse duration. We consider a 20-opticalcycle laser pulse with a peak intensity $I_{0}=5 \times 10^{13} \mathrm{~W} / \mathrm{cm}^{2}$, a wavelength $\lambda=800 \mathrm{~nm}$, and $\alpha=0$. In this high-precision calculation, $k_{\max }$ is taken to be $10^{4}$ a.u., $\gamma=2, l_{\max }=15$, and 2000 grid points are employed. In the propagation, the number of eigenfunctions involved can be reduced based on the maximum energy of electrons during the process. Only the eigenfunctions whose eigenenergies are less than the maximum energy (e.g., $40 U_{P}$ ) are included in the calculation. This truncation greatly reduces the number of eigenfunctions in the propagation and speeds up the calculation. The convergence is inspected by checking both the normalization constant and ATI spectra.

In Fig. 1, we show the high-resolution color contour of the DDIP. The radial distance and the angle denote the photoelectron energy and direction with respect to the laser field, respectively. The color density is plotted in logarithmic scale and represents the values of the DDIP. The photoelectron energy-angular distribution displays complex interference patterns. It consists of a series of rings with fine structures. The rings represent the angular distribution of the photoelectron

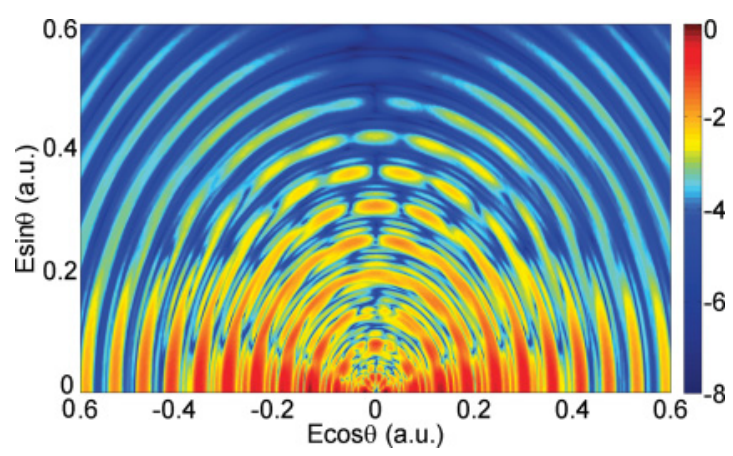

FIG. 1. (Color online) Photoelectron energy-angular distribution (DDIP) for the 20-optical-cycle laser pulse with a peak intensity of $5 \times 10^{13} \mathrm{~W} / \mathrm{cm}^{2}$ and a wavelength of $800 \mathrm{~nm}$.

ATI peaks. The spacing of the adjacent rings equals to the photon energy. The first ring corresponds to the angular distribution of the first ATI peak. It comprises several radial stripes spreading out from the center. These stripes are induced by the long-range Coulomb potential and are related to the fact that the ATI peak is determined by one dominant partial wave in the final state $[15,17]$. The number of the stripes equals the angular momentum quantum number of the dominant partial wave in the final state plus one $[15,17,18,63]$. In Fig. 1, the first ring contains six stripes. Thus the dominant final state for the first ATI peak has the angular momentum quantum number of 5. The pattern of the energy-angular distribution and the stripe number of the first ring are in good agreement with those in the literature $[15,18,63]$. As for the fine structures, one kind of explanation is that they are produced by the rapidly changing ponderomotive potential in the short laser pulse [16]. Another explanation, which is suitable for both short and long pulses, is that the fine structures are induced by the coherence of the two contributions from the leading and trailing edges of the pulse envelope $[18,64]$. Furthermore, Fig. 1 shows that the photoelectron energy-angular distribution is primarily ring shaped. This indicates that the photoelectron distribution does not depend strongly on the direction, and the photoelectron is emitted isotropically in the multiphoton ionization when $\gamma>1$.

In Fig. 2, we show the photoelectron ATI spectra in a solid curve and we show the whole spectra in logarithmic scale in the inset. The energy spectra display the typical ATI spectra structures: The peaks are separated by the photon energy; the first cutoff occurs at $2 U_{P}$ for the direct ionization, and the second cutoff occurs at $10 U_{P}$ for the rescattering. Each ATI peak is accompanied by several subpeaks on the right-hand side. These subpeaks correspond to the fine structures in Fig. 1 and again are produced by the coherence of the two contributions from the leading and trailing edges of the pulse envelope $[18,64]$. For direct comparison, we also show the ATI spectra of Ref. [18] in the dashed-dotted curve in Fig. 2. It is shown that the structure and substructure of the ATI spectra as well as the ATI peak positions and heights obtained in our calculation are in good agreement with those in Ref. [18].

\section{Above-threshold ionization of hydrogen atom in intense short-wavelength laser fields}

As an application of the proposed approach, we apply the method to the nonperturbative study of multiphoton ATI 


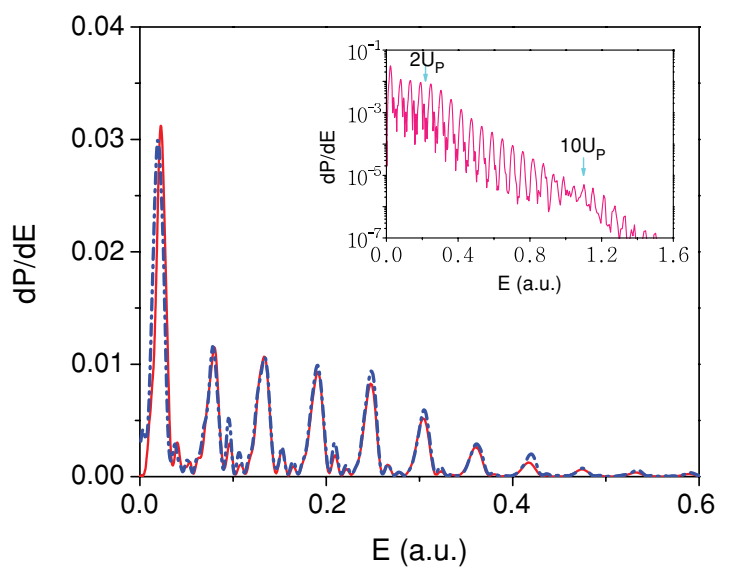

FIG. 2. (Color online) Photoelectron ATI spectra for the 20-optical-cycle laser pulse with a peak intensity of $5 \times 10^{13} \mathrm{~W} / \mathrm{cm}^{2}$ and a wavelength of $800 \mathrm{~nm}$. The solid curve is the result of present work and the dashed-dotted curve is the result of Ref. [18]. The inset is the ATI spectra plotted in logarithmic scales.

of atomic hydrogen in the presence of a 10-optical-cycle short-wavelength $\lambda=91.13 \mathrm{~nm}$ laser pulse. The pulse shape is the same as that used in the previous subsection and is given by Eq. (34). The angular frequency of this laser is $\omega=0.5$ a.u. The dipole approximation is expected to be a good approximation when the laser intensity is not very high. In Figs. 3(a)-3(d), the high-resolution color contours of the photoelectron energy-angular distribution are plotted for the laser fields with $\varepsilon_{0}=0.3,0.5,1.0$, and 1.5 a.u., respectively. As in Fig. 1, the radial distance and angle represent the photoelectron energy and motion direction. The color density is plotted in logarithmic scale and characterizes the value of the DDIP.

It is shown that the energy-angular distribution consists of a series of stripes with equal spacing. For the lower laser intensity when $\gamma \gtrsim 1$ in Figs. 3(a) and 3(b), the primary stripes are ring shaped. The photoelectron distribution is close to circular and almost does not depend on the direction. This again demonstrates that the photoelectron angular distribution is nearly independent of the direction in the multiphoton ionization. However, for the higher laser intensity when $\gamma \ll 1$ in Figs. 3(c) and 3(d), the stripes are no longer ring shaped, particularly for the high-energy photoelectrons. The photoelectron distribution is dumbbell shaped and thus strongly depends on the direction. Such a kind of photoelectron energy-angular distribution has been observed in the recent experiment [30]. As expected, the photoelectron angular distribution expands with the increase of the laser intensity. However, the distribution in the direction parallel to the laser field magnifies faster than in the direction perpendicular to the laser field and thus is squeezed along the laser field direction. This phenomenon is also found in the hydrogen molecule driven by long-wavelength intense laser fields [65]. It seems to be a typical feature of the photoelectron energy-angular distribution in the tunneling ionization. Moreover, in all the cases here, the first ring has two stripes. Thus the angular momentum quantum number of the dominant final state for the first peak is $l=1$. This is not surprising. For the laser frequency used in the calculation, the ionization threshold is lowered [62] and the ground-state electron with $l=0$ can be ionized by absorbing one electron, making the electron in the final state having $l=1$.

The high-resolution photoelectron ATI spectra are displayed in Figs. 4(a)-4(d) for the laser fields with $\varepsilon_{0}=0.3$, $0.5,1.0$, and 1.5 a.u., respectively. For all the cases, from the multiphoton to tunneling ionization shown in these figures, the ATI spectra exhibit well-resolved peaks with equal spacing. Although the ionization threshold is lowered for the laser fields used [62], the shift is quite small. Thus the ATI peaks do not shift obviously [21]. However, owing to the interference $[18,64]$ there are several subpeaks followed by each ATI peak.
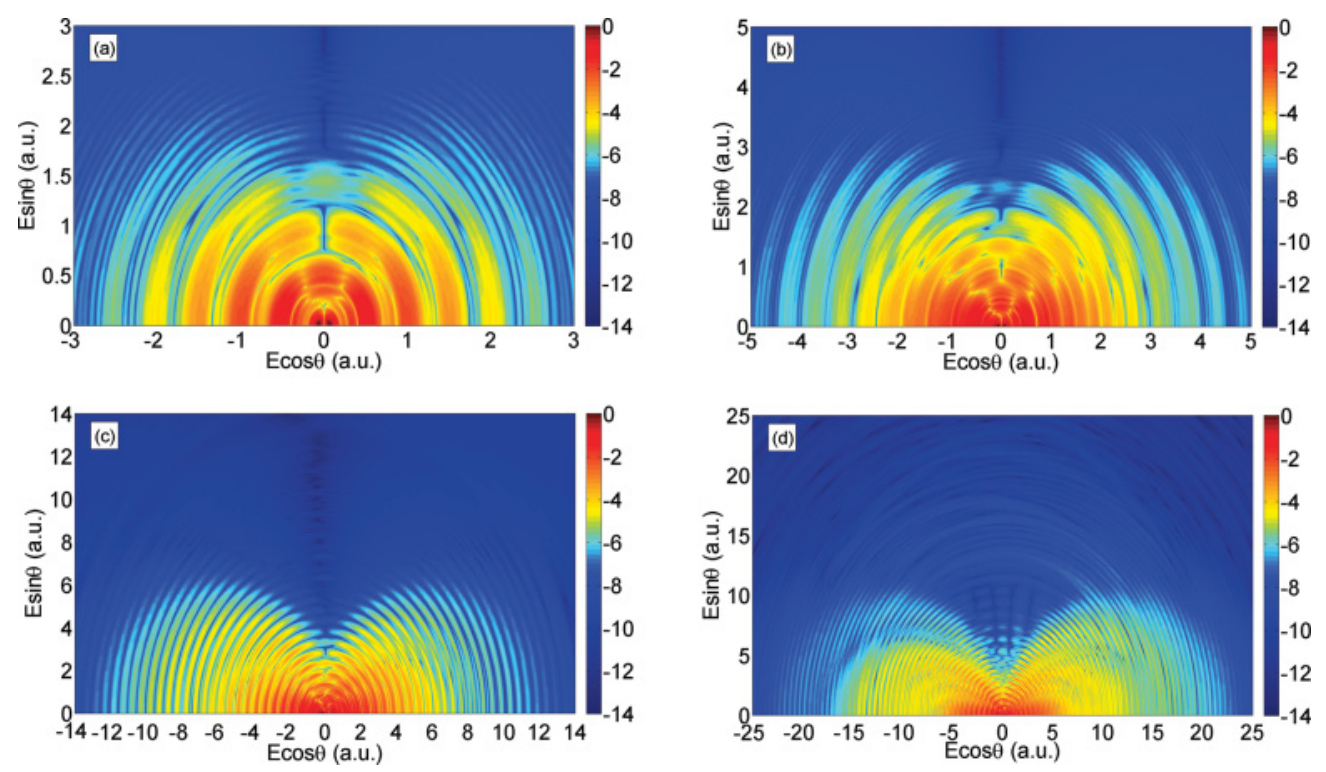

FIG. 3. (Color online) Photoelectron energy-angular distribution (DDIP) for the 10-optical-cycle laser pulse with a wavelength of $91.13 \mathrm{~nm}$ and different peak intensities: (a) $\varepsilon_{0}=0.3$ a.u., (b) $\varepsilon_{0}=0.5$ a.u., (c) $\varepsilon_{0}=1.0$ a.u., and (d) $\varepsilon_{0}=1.5$ a.u. 

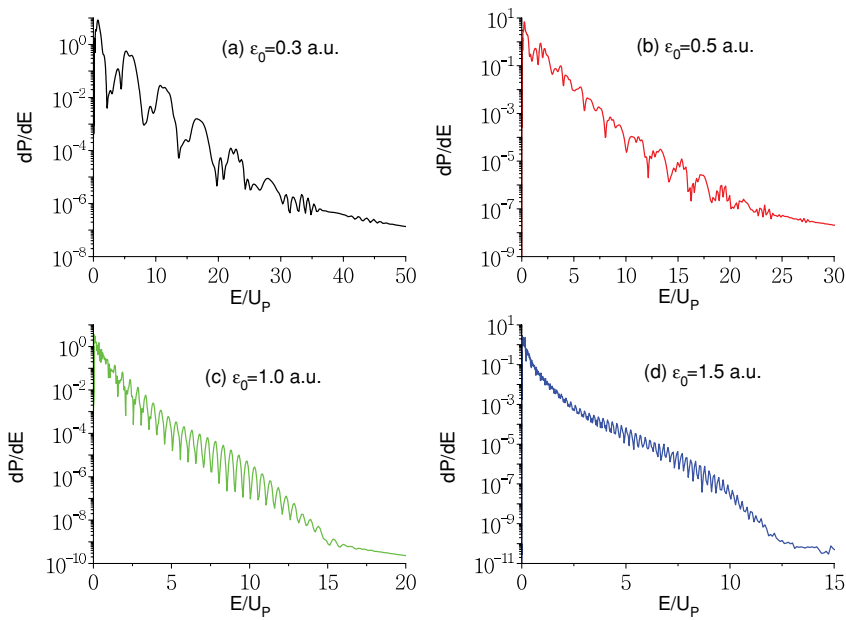

FIG. 4. (Color online) Photoelectron ATI spectra (DIP) for the 10-optical-cycle laser pulse with a wavelength of $91.13 \mathrm{~nm}$ and different peak intensities: (a) $\varepsilon_{0}=0.3$ a.u., (b) $\varepsilon_{0}=0.5$ a.u., (c) $\varepsilon_{0}=1.0$ a.u., and (d) $\varepsilon_{0}=1.5$ a.u.

The fine structures are particularly pronounced for the first several ATI peaks. Owing to the fine structures, the first several ATI peaks become blurred. In addition, the ratio of the maximum photoelectron energy to the ponderomotive energy, $E_{\max } / U_{P}$, changes with the laser intensity rather than remains at a constant 10 . The lower the laser intensity, the larger the ratio.

In order to investigate the relation of the maximum photoelectron energy $E_{\max }$ and laser field $\varepsilon_{0}$, we plot the scaled maximum photoelectron momentum defined by $\beta=$ $\sqrt{E_{\max } / U_{P}}$ vs $1 / \varepsilon_{0}$ in Fig. 5. The open circles are the numerical results and the line is the result of linear fitting. It is shown that $\beta$ increases linearly with $1 / \varepsilon_{0}$. The relation between them can be well described by

$$
\beta=2.712+\frac{1.152}{\varepsilon_{0}} .
$$

This relation suggests that at the lower laser intensity, the maximum photoelectron energy can be much larger than

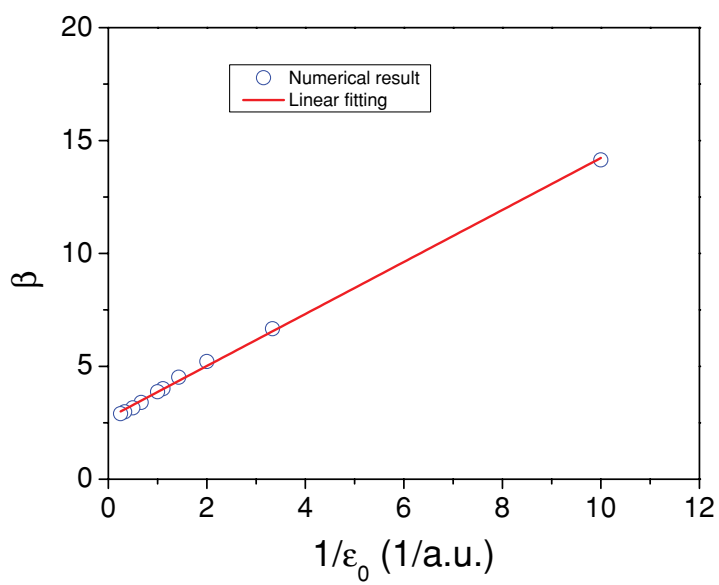

FIG. 5. (Color online) The scaled maximum photoelectron momentum $\beta$ vs $1 / \varepsilon_{0}$ for the 10-optical-cycle laser pulse with a wavelength of $91.13 \mathrm{~nm}$.

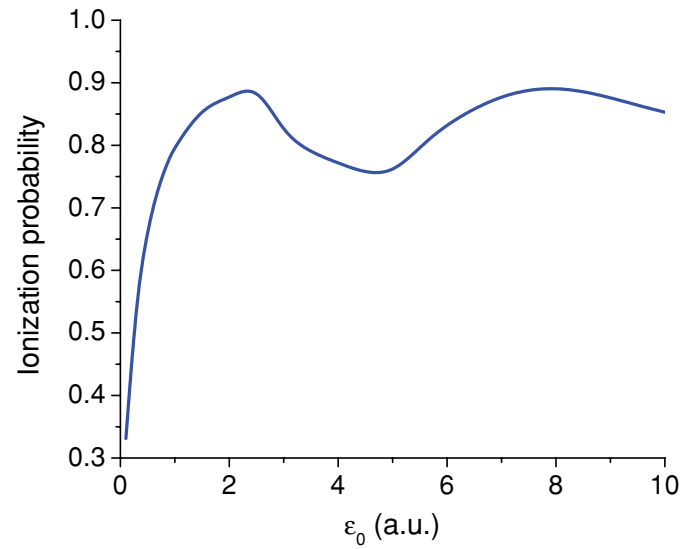

FIG. 6. (Color online) Ionization probability vs laser field amplitude $\varepsilon_{0}$ for the 10-optical-cycle pulse with a wavelength of $91.13 \mathrm{~nm}$.

$10 U_{P}$, while at the higher laser intensity, the maximum energy is smaller than $10 U_{P}$. In the high-intensity limit, the maximum energy is $\sim 7.35 U_{P}$. This is totally different from the classical prediction that the maximum photoelectron energy is $10 U_{P}$. Currently we do not have a reasonable theoretical interpretation to this empirical relation.

In Fig. 6, the total ionization probability is plotted versus the amplitude of the laser field $\varepsilon_{0}$. It is shown that the ionization probability increases with $\varepsilon_{0}$ when $\varepsilon_{0} \leqslant 2.5$ a.u., decreases with $\varepsilon_{0}$ when $2.5<\varepsilon_{0} \leqslant 5$ a.u., increases with $\varepsilon_{0}$ when $5<$ $\varepsilon_{0} \leqslant 8$ a.u., and decreases with $\varepsilon_{0}$ when $\varepsilon_{0}>8$ a.u. During this process, the ionization rates decrease with $\varepsilon_{0}$ when $2.5<$ $\varepsilon_{0} \leqslant 5$ a.u. and $\varepsilon_{0}>8$ a.u. This is the well-known strong-field atomic stabilization phenomenon [66-72]. This phenomenon can occur at any frequency and can be enhanced by relativistic effects [70]. Detailed discussions about the strong-field atomic stabilization and various physical mechanisms have been given in recent review papers $[71,72]$.

In summary, based on the fact that the momentum of an electron is always finite in any physical process and the wave function of the electron is zero when its momentum is greater than a certain maximum value, we have developed an efficient and accurate $\mathcal{P}$-space computational approach for the study of ionization dynamics of atomic systems driven by intense laser fields. This approach has the following features and advantages. (a) Because the basic equation, the $\mathcal{P}$-space TDSE, is obtained from the Fourier transform of the $\mathcal{R}$-space TDSE in the whole $\mathcal{R}$ space, this approach is free of boundary reflection and contains all the information related to the continuum-state physical processes. (b) The wave function is computed in a finite $\mathcal{P}$-space volume under the zero boundary condition by solving the $\mathcal{P}$-space TDSE. This makes the computation simpler and more efficient. (c) The GPS method and mapping techniques are extended to the $\mathcal{P}$-space calculations. This allows for the nonuniform and optimal $\mathcal{P}$-space discretization with the use of only a modest number of grid points to achieve the accurate wave function. (d) Because the unperturbed Hamiltonian and laser-atom interaction operator transform the wave function within two different subspaces, respectively, the calculation of the time-dependent wave function using the split-operator time propagators is more competent and 
economical. (e) Because the $\mathcal{P}$-space wave function represents the probability amplitude of an electron having the momentum $\mathbf{k}$, the differential ionization probabilities are calculated directly from the continuum-state wave function that is acquired from the total electron wave function with the help of the continuum-state projection operator. The continuum-state projection operator is constructed by the eigenfunctions of the unperturbed Hamiltonian with positive eigenenergies. For test of the method, we first apply the approach to the calculation of the multiphoton ionization rates, photoelectron energy-angular distribution, and ATI spectra of a hydrogen atom exposed to the intense laser fields. The results are in good agreement with those from other $\mathcal{R}$-space calculations. We then extend the $\mathcal{P}$-space approach to the study of the multiphoton ATI of the hydrogen atom in the presence of the intense short-wavelength laser fields, where the $\mathcal{R}$-space methods are more difficult to achieve the convergence because the electron may go very far away from the nucleus. The high-resolution photoelectron ATI spectra and energy-angular distribution have been achieved by the present approach. We find that with the increase of the laser intensity, the ATI regime shifts from multiphoton ionization to tunneling ionization, and accordingly, the photoelectron energy-angular distribution changes from circular to dumbbell shaped and is squeezed along the laser field direction. We also find that the ratio of the maximum photoelectron energy to the ponderomotive energy $U_{P}$ varies with the laser field. For the lower laser intensity, the maximum energy is larger than $10 U_{P}$, and for the higher laser intensity the maximum energy is smaller than $10 U_{P}$. An empirical expression has been obtained to depict the relation of the maximum photoelectron energy and laser field strength. Finally, the total ionization probabilities are calculated for different laser field amplitudes and the strong-field atomic stabilization is reproduced.

\section{ACKNOWLEDGMENTS}

This work was partially supported by the Chemical Sciences, Geosciences, and Biosciences Division of the Office of Basic Energy Sciences, Office of Sciences, US Department of Energy and by the US National Science Foundation. We would like to acknowledge also the partial support of National Science Council of Taiwan (Grant No. 97-2112-M-002-003MY3) and National Taiwan University (Grants No. 98R0045 and No. 99R80870).
[1] R. R. Freeman and P. H. Bucksbaum, J. Phys. B 24, 325 (1991).

[2] W. Becker, F. Grasbon, R. Kopold, D. B. Milošvić, G. G. Paulus, and H. Walther, Adv. At. Mol. Opt. Phys. 48, 35 (2002).

[3] P. Agostini, F. Fabre, G. Mainfray, G. Petite, and N. K. Rahman, Phys. Rev. Lett. 42, 1127 (1979).

[4] T. Brabec and F. Krausz, Rev. Mod. Phys. 72, 545 (2000).

[5] F. Krausz and M. Ivanov, Rev. Mod. Phys. 81, 163 (2009).

[6] L. V. Keldysh, J. Exp. Theor. Phys. (USSR) 47, 1945 (1964) [Sov. Phys. JETP 20, 1307 (1965)].

[7] B. Yang, K. J. Schafer, B. Walker, K. C. Kulander, P. Agostini, and L. F. DiMauro, Phys. Rev. Lett. 71, 3770 (1993).

[8] G. G. Paulus, W. Becker, W. Nicklich, and H. Walther, J. Phys. B 27, L703 (1994).

[9] M. Lewenstein, K. C. Kulander, K. J. Schafer, and P. H. Bucksbaum, Phys. Rev. A 51, 1495 (1995).

[10] J. Z. Kamiński, A. Jaroń, and F. Ehlotzky, Phys. Rev. A 53, 1756 (1996).

[11] K. J. Schafer and K. C. Kulander, Phys. Rev. A 42, 5794 (1990).

[12] M. B. Gaarde, K. J. Schafer, K. C. Kulander, B. Sheehy, D. Kim, and L. F. DiMauro, Phys. Rev. Lett. 84, 2822 (2000).

[13] J. Zhang, W. Zhang, Z. Xu, X. Li, P. Fu, D.-S. Guo, and R. R. Freeman, J. Phys. B 35, 4809 (2002).

[14] R. Wiehle, B. Witzel, H. Helm, and E. Cormier, Phys. Rev. A 67, 063405 (2003).

[15] D. G. Arbó, S. Yoshida, E. Persson, K. I. Dimitriou, and J. Burgdörfer, Phys. Rev. Lett. 96, 143003 (2006).

[16] M. Wickenhauser, X. M. Tong, and C. D. Lin, Phys. Rev. A 73, 011401(R) (2006).

[17] Z. Chen, T. Morishita, A.-T. Le, M. Wickenhauser, X. M. Tong, and C. D. Lin, Phys. Rev. A 74, 053405 (2006).

[18] D. A. Telnov and Shih-I Chu, Phys. Rev. A 79, 043421 (2009).

[19] P. Kruit, J. Kimman, H. G. Muller, and M. J. van der Wiel, Phys. Rev. A 28, 248 (1983).

[20] T. J. McIlrath, P. H. Bucksbaum, R. R. Freeman, and M. Bashkansky, Phys. Rev. A 35, 4611 (1987).
[21] R. R. Freeman, P. H. Bucksbaum, H. Milchberg, S. Darack, D. Schumacher, and M. E. Geusic, Phys. Rev. Lett. 59, 1092 (1987).

[22] P. B. Corkum, N. H. Burnett, and F. Brunel, Phys. Rev. Lett. 62, 1259 (1989).

[23] G. G. Paulus, W. Nicklich, H. Xu, P. Lambropoulos, and H. Walther, Phys. Rev. Lett. 72, 2851 (1994).

[24] H. Rottke, B. Wolff-Rottke, D. Feldmann, K. H. Welge, M. Dörr, R. M. Potvliege, and R. Shakeshaft, Phys. Rev. A 49, 4837 (1994).

[25] G. G. Paulus, F. Grasbon, H. Walther, P. Villoresi, M. Nisoli, S. Stagira, E. Priori, and S. De Silvestri, Nature (London) 414, 182 (2001).

[26] G. G. Paulus, F. Lindner, H. Walther, A. Baltuska, E. Goulielmakis, M. Lezius, and F. Krausz, Phys. Rev. Lett. 91, 253004 (2003).

[27] A. Rudenko, K. Zrost, C. D. Schröter, V. L. B. de Jesus, B. Feuerstein, R. Moshammer, and J. Ullrich, J. Phys. B 37, L407 (2004).

[28] A. Rudenko, K. Zrost, T. Ergler, A. B. Voitkiv, B. Najjari, V. L. B. de Jesus, B. Feuerstein, C. D. Schröter, R. Moshammer, and J. Ullrich, J. Phys. B 38, L191 (2005).

[29] C. M. Maharjan, A. S. Alnaser, I. Litvinyuk, P. Ranitovic, and C. L. Cocke, J. Phys. B 39, 1955 (2006).

[30] D. Ray, B. Ulrich, I. Bocharova, C. Maharjan, P. Ranitovic, B. Gramkow, M. Magrakvelidze, S. De, I. V. Litvinyuk, A. T. Le et al., Phys. Rev. Lett. 100, 143002 (2008).

[31] C. I. Blaga, F. Catoire, P. Colosimo, G. G. Paulus, H. G. Muller, P. Agostini, and L. F. DiMauro, Nat. Phys. 5, 335 (2009).

[32] L.-H. Yu, M. Babzien, I. Ben-Zvi, L. F. DiMauro, A. Doyuran, W. Graves, E. Johnson, S. Krinsky, R. Malone, I. Pogorelsky et al., Science 289, 932 (2000).

[33] G. R. Neil and L. Merminga, Rev. Mod. Phys. 74, 685 (2002).

[34] J. Feldhaus, J. Arthur, and J. B. Hastings, J. Phys. B 38, S799 (2005). 
[35] W. Li, R. R. Lucchese, A. Doyuran, Z. Wu, H. Loos, G. E. Hall, and A. G. Suits, Phys. Rev. Lett. 92, 083002 (2004).

[36] A. A. Sorokin, S. V. Bobashev, K. Tiedtke, and M. Richter, J. Phys. B 39, L299 (2006).

[37] N. Rohringer and R. Santra, Phys. Rev. A 76, 033416 (2007).

[38] G. Yao and S.-I. Chu, J. Phys. B 25, 363 (1992).

[39] U.-L. Pen and T. F. Jiang, Phys. Rev. A 46, 4297 (1992).

[40] T. F. Jiang, Comput. Phys. Commun. 178, 571 (2008).

[41] Z. Zhou and S.-I. Chu, Europhys. Lett. 88, 17008 (2009).

[42] G. Yao and S.-I. Chu, Chem. Phys. Lett. 204, 381 (1993).

[43] J. Wang, Shih-I Chu, and C. Laughlin, Phys. Rev. A 50, 3208 (1994).

[44] X. M. Tong and S. I. Chu, Chem. Phys. 217, 119 (1997).

[45] Z. Zhou and Shih-I Chu, Phys. Rev. A 71, 022513 (2005).

[46] R. H. Landau, Phys. Rev. C 27, 2191 (1983).

[47] K. M. Maung, D. E. Kahana, and J. W. Norbury, Phys. Rev. D 47, 1182 (1993).

[48] J. W. Norbury, K. M. Maung, and D. E. Kahana, Phys. Rev. A 50, 2075 (1994).

[49] Y. RaeKwon and F. Tabakin, Phys. Rev. C 18, 932 (1978).

[50] I. A. Ivanov and J. Mitroy, Comput. Phys. Commun. 134, 317 (2001).

[51] H. A. Bethe and E. E. Salpeter, Quantum Mechanics of Oneand Two-Electron Atoms (Plenum, New York, 1977).

[52] J. W. Norbury, K. M. Maung, and D. E. Kahana, Phys. Rev. A 50, 3609 (1994).

[53] E. Cormier and P. Lambropoulos, J. Phys. B 30, 77 (1997).

[54] T. Birkeland, M. Frre, J. P. Hansen, and S. Selst, J. Phys. B 37, 4205 (2004).
[55] We have extended the conventional method to the P-space calculation and obtained the results in very good agreement with those presented in this work.

[56] M. Awasthi, Y. V. Vanne, A. Saenz, A. Castro, and P. Decleva, Phys. Rev. A 77, 063403 (2008).

[57] M. Abu-samha and L. B. Madsen, Phys. Rev. A 81, 033416 (2010).

[58] S. Mallampalli and J. Sapirstein, J. Phys. B 31, 3779 (1998).

[59] P. T. Greenland, Nature (London) 387, 548 (1997).

[60] S. R. Wilkinson, C. F. Gharucha, M. C. Fischer, K. W. Madison, P. R. Morrow, Q. Niu, B. Sundaram, and M. G. Raizen, Nature (London) 387, 575 (1997).

[61] K. C. Kulander, Phys. Rev. A 35, 445 (1987).

[62] Shih-I Chu and J. Cooper, Phys. Rev. A 32, 2769 (1985).

[63] D. G. Arbó, J. E. Miraglia, M. S. Gravielle, K. Schiessl, E. Persson, and J. Burgdörfer, Phys. Rev. A 77, 013401 (2008).

[64] D. A. Telnov and S. I. Chu, J. Phys. B 28, 2407 (1995).

[65] Z. Zhou and S. I. Chu (submitted to Phys. Rev. A 2010).

[66] M. Pont and M. Gavrila, Phys. Rev. Lett. 65, 2362 (1990).

[67] K. C. Kulander, K. J. Schafer, and J. L. Krause, Phys. Rev. Lett. 66, 2601 (1991).

[68] M. Dörr, R. M. Potvliege, D. Proulx, and R. Shakeshaft, Phys. Rev. A 43, 3729 (1991).

[69] K. Burnett, V. C. Reed, and P. L. Knight, J. Phys. B 26, 561 (1993).

[70] H. R. Reiss, Opt. Express 8, 99 (2001).

[71] M. Gavrila, J. Phys. B 35, R147 (2002).

[72] A. M. Popov, O. V. Tikhonova, and E. A. Volkova, J. Phys. B 36, R125 (2003). 